\title{
A TRANSCENDENCE MEASURE FOR SOME SPECIAL VALUES OF ELLIPTIC FUNCTIONS
}

\begin{abstract}
ROBERT TUBBS
AbStract. Among T. Schneider's results is the following: Let $\wp(z)$ be the Weierstrass elliptic function with algebraic invariants. If $\wp(u)$ and $\beta$ are both algebraic, $\beta \notin K_{\tau}$, then $\wp(\beta u)$ is transcendental. In this paper we provide a transcendence measure for this value.

Let $P(X)$ be a nonzero polynomial, with integral coefficients, of degree $d$ and height $h$, and put $t=d+\log h$. Then there is an effectively computable constant $C$, which does not depend on $P(X)$, such that:

(A) If $\wp(z)$ has complex multiplication then $\log |P(\wp(\beta u))|>-C d^{2} t^{2}(\log t)^{4}$.

(B) If $\wp(z)$ does not have complex multiplication then $\log |P(\wp(\beta u))|>$ $-C d^{6} t^{2}(\log t)^{14}$.
\end{abstract}

Introduction. The aim of this paper is to provide transcendence measures for the Weierstrass $\wp$-function evaluated at numbers from a particular class of values. We derive our measures under the assumption that the invariants for $\ell(z)$ are algebraic integers. In particular, we prove the following result.

THEOREM. Suppose that $u$ is a nontorsion algebraic point of $\wp(z)$ and $\beta$ is an algebraic number, $\beta \notin K_{\tau}$. Let $P(X)$ be a nonzero integral polynomial with $d=\operatorname{deg} P$, $h=\mathrm{ht} P$, and $t=d+\log h$. Then there is an effectively computable constant $C$ (depending only on $u, \beta, \wp)$ such that:

(A) If $\wp(z)$ has complex multiplication then

$$
\log |P(\wp(\beta u))|>-C d^{2} t^{2}(\log t)^{4} .
$$

(B) If $\wp(z)$ does not have complex multiplication then

$$
\log |P(\wp(\beta u))|>-C d^{6} t^{2}(\log t)^{14} .
$$

Preliminaries. We take $\omega_{1}, \omega_{2}$ for the generators of the period lattice $\Omega$ of $\ell(z)$, that is, $\Omega=Z \omega_{1}+Z \omega_{2} ; \wp$ is periodic with respect to $\Omega$ and is meromorphic with poles on $\Omega$. If we take $g_{2}=\sum_{\omega \in \Omega^{*}} \omega^{-4}$ and $g_{3}=\sum_{\omega \in \Omega^{*}} \omega^{-6}$, where $\Omega^{*}=\Omega-\{0\}$, then $\wp$ satisfies the differential equation $\left(\wp^{\prime}(z)\right)^{2}=4 \wp^{3}(z)-g_{2} \wp(z)-g_{3}$. The numbers $g_{2}$ and $g_{3}$ are called the invariants of $\wp$. Throughout this paper we assume that $g_{2} / 4$ and $g_{3} / 4$ are algebraic integers.

We recall that $\wp$ is said to have complex multiplication when $\tau=\omega_{1} / \omega_{2}$ is algebraic, in which case $\tau$ is a quadratic irrationality. In this situation the mapping

Received by the editors April 3, 1982.

1980 Mathematics Subject Classification. Primary 10F35.

Key words and phrases. Transcendental numbers, transcendence measure, elliptic functions. 
$\sigma: \wp(z) \rightarrow \wp(\sigma z)$ is an endomorphism of $\mathbf{Q} / \Omega$ for all $\sigma \in \theta . \theta=Z+Z \tau$ is the set of multiplications of $\wp$. We also use the notation

$$
\Theta(S)=\left\{\sigma \in O \mid \sigma=s_{1}+s_{2} \tau \text { with }\left|s_{i}\right| \leqslant S \text { for } i=1,2\right\} \text {. }
$$

When $\tau$ is not algebraic then $\theta=Z$ and $\theta(S)$ consists of integers $\sigma$ with $|\sigma| \leqslant S$. In the case of complex multiplication we put $K_{\tau}=\mathbf{Q}(\tau)$, otherwise $K_{\tau}=\mathbf{Q}$.

It is a result of T. Schneider that for $\beta \notin K_{\tau}$ the functions $\wp(z)$ and $\wp^{*}(z)=\wp(\beta z)$ satisfy not all of $g_{2}, g_{3}, g_{2}^{*}, g_{3}^{*}, \beta, \wp(u)$, and $\wp^{*}(u)$ are algebraic, where we have let $g_{2}^{*}$ and $g_{3}^{*}$ denote the invariants of $\wp^{*}(z)$. From our hypothesis that $\beta$ and $\wp(u)$ are algebraic, and $\beta \notin K_{\tau}$, it follows that $\wp(\beta u)$ is transcendental.

Our method of proof, due to A. O. Gelfond, will require that we define parameters $D, K$, and $S$ and then construct a polynomial $P^{*}(X, Y)$ of total degree at most $D$ such that the auxiliary function $\Phi(z)=P^{*}(\wp(z), \wp(\beta z))$ satisfies $\Phi^{(k)}(\sigma u)=0$ for $0 \leqslant k \leqslant K$ and $\sigma \in \mathcal{O}(S)$. Our construction of the polynomial $P^{*}(X, Y)$ requires the following lemma.

LEMMA 1. Let $S$ and $R$ be rational integers and $F$ a number field. Consider the system of equations $\sum_{i=1}^{S} a_{i j} z_{i}=0$ for $1 \leqslant j \leqslant R$, where $a_{i j}$ satisfy (i) $a_{i j} \in$ $F\left[X_{1}, \ldots, X_{6}\right]$ with coefficients which are integers in $F$ with sizes bounded by $A$, and (ii) $\operatorname{deg}_{X_{k}} a_{i j} \leqslant d_{k} \quad(1 \leqslant k \leqslant 6)$ with $1=d_{4}=d_{5}=d_{6}$. Then, provided that $S \leqslant$ $[F: \mathbf{Q}] 2^{6} R$, the system has a nonzero solution $\left(z_{1}, \ldots, z_{S}\right)$ with $z_{i} \in Z\left[X_{1}, X_{2}, X_{3}\right]$ with $\operatorname{deg}_{X_{k}} z_{i} \leqslant d_{k}(k=1,2,3)$ and

$$
\operatorname{ht}\left(z_{i}\right) \leqslant\left(A S \prod_{k=1}^{3}\left(1+d_{k}\right)^{2}\right)^{[F: \mathbf{Q}] 2^{6} R /\left(S-[F: \mathbf{Q}] 2^{6} R\right)} .
$$

Proof. Substitute $z_{i}$ into each equation, leaving the coefficients of $z_{i}$ undetermined. If we then multiply out these expressions and collect together the coefficients of each monomial $X_{4}^{e(4)} X_{5}^{e(5)} X_{6}^{e(6)}, e(k) \in\{0,1\}$, we obtain eight polynomials $P_{e(4), e(5), e(6)}\left(X_{1}, X_{2}, X_{3}\right)$. Each of these polynomials contain $\Pi_{k=1}^{3}\left(1+2 d_{k}\right)$ monomials with coefficients in terms of $S \prod_{k=1}^{3}\left(1+d_{k}\right)$ unknowns (these are the "undetermined coefficients" of the $z_{i}$ ). Setting the coefficient of each of the monomials equal to zero yields $8 \Pi_{k=1}^{3}\left(1+2 d_{k}\right)$ equations in $S \prod_{k=1}^{3}\left(1+d_{k}\right)$ unknowns. These equations have coefficients which are algebraic integers of size bounded by $A \Pi_{k=1}^{3}\left(1+d_{k}\right)$. Therefore by the standard Thue-Siegel lemma [5, Lemma 1.3.2] we can solve over the rational integers provided that

$$
S \prod_{k=1}^{3}\left(1+d_{k}\right) \leqslant[F: \mathbf{Q}] 8 \prod_{k=1}^{3}\left(1+2 d_{k}\right) .
$$

It suffices that $S \leqslant[F: \mathbf{Q}] 2^{6} R$. Then the absolute value of the solutions, which are the coefficients of the polynomials $z_{i}$, are majorized by the bound given above.

In the next lemma we take $\sigma(z)$ to be the Weierstrass sigma function. We recall that $\sigma(z)$ is defined with respect to the lattice $\Omega$ so that $\sigma(z)$ is entire with zeros of order 1 at each lattice point. It is then basic that $\sigma^{2}(z) \wp(z)$ and $\sigma^{3}(z) \wp^{\prime}(z)$ are entire functions. 
LEMMA 2. Let $u$ be a nontorsion point for $\wp(z)$ and $\alpha_{1}, \ldots, \alpha_{n} K_{\tau}$-linearly independent numbers. There exists a constant $C_{0}$ (depending only on $u, \alpha_{1}, \ldots, \alpha_{n}$ ) such that the set

$$
\theta^{\prime}(s)=\left\{s \in \mathcal{O}(S)|| \sigma\left(s \alpha_{i} u\right) \mid \geqslant C_{0} e^{-S^{2}} \text { for all } i=1, \ldots, n\right\}
$$

satisfies card $\Theta^{\prime}(S) \geqslant(n+1)^{-1}$ card $\mathfrak{C}^{(}(S)$.

Proof. We consider separately the cases when $\wp$ does or does not have complex multiplication.

If $\wp(z)$ does not have complex multiplication we let $t=n+2$ and define a constant $c^{\prime}$ by

$$
c^{\prime}=\min \left\{\left|s \alpha_{i} u-\omega\right| 1 \leqslant i \leqslant n, \omega \in \Omega, s \in \mathfrak{C}(2 t)\right\} .
$$

For each $p \in N$ we let $R_{p}=\{r \mid p t \leqslant r<(p+1) t\}$. Then $\mathcal{E}(S)$ is contained in the disjoint union of the sets $R_{p}$ for $0 \leqslant p \leqslant S n^{-1}$. We begin by showing that each set $R_{p}$ contains at most $n$ multiplications $s$ such that $\left|s \alpha_{i} u-\omega\right|<\frac{1}{2} c^{\prime}$ for some $1 \leqslant i \leqslant n$ and some $\omega \in \Omega$. This follows since if there are at least $n+1$ such multiplications, then two of them, say $s_{1}$ and $s_{2}$, correspond to the same $\alpha_{i}$. In this case $s_{0}=s_{1}-s_{2}$ lies in $\Theta(2 t)$ and contradicts our choice of $c^{\prime}$.

Therefore at least one of the multiplications in each $R_{p}$ satisfies $\left|s \alpha_{i} u-\omega\right|>\frac{1}{2} c^{\prime}$ for all $i$ and for all $\omega \in \Omega$. Yet $p \leqslant(n+1)^{-1} S$ implies that $R_{p} \subseteq \mathcal{O}(S)$ and therefore $\rho(S)$ contains at least $(n+1)^{-1} S$ of these multiplications.

In this situation where $\wp$ has complex multiplication we take $t=[\sqrt{n+1}]$ and define $c^{\prime \prime}$ as we defined $c^{\prime}$ above. For each pair of integers $p, q$ we let $R_{p, q}=$ $\{r+s \tau \mid p t \leqslant r<(p+1) t, q t \leqslant s<(q+1) t\}$. Arguing as above we see that at least $(n+1)^{-1} S^{2}$ of the multiplications $s$ in $\Theta(S)$ satisfy $\left|s \alpha_{i} u-\omega\right|>\frac{1}{2} c^{\prime \prime}$ for all $i$ and all $\omega \in \Omega$.

In either case let $C_{0}=\min \left\{c^{\prime}, c^{\prime \prime}\right\}$ and apply Lemma 7.1 of [4] to obtain the desired result.

Once we have constructed our auxiliary function with zeros of order $K$ at the points $s u$ for $s \in \mathcal{O}^{\prime}(S)$, we will need an estimate for the total order of zeros at these points. This estimate is provided by the following result of Brownawell and Masser.

LeMma 3. For a nonnegative integer $N$ let $\delta_{0}, \ldots, \delta_{N}$ be complex numbers and let $z_{0}, \ldots, z_{N}$ be points which are mutually incongruent modulo $\Omega$. Suppose that $\wp(z)$ and $\wp\left(a z+\delta_{i}\right)$ are analytic at $z=z_{i}$ for some nonzero a and for each $i, 0 \leqslant i \leqslant N$. Then for $P(X, Y) \in C[X, Y]$, a nonzero polynomial with $\operatorname{deg}_{X} \leqslant L$ and $\operatorname{deg}_{Y} \leqslant M$, such that the functions $\Theta_{i}(z)=P\left(\wp(z), \wp\left(a z+\delta_{i}\right)\right)(0 \leqslant i \leqslant N)$ are not identically zero, we have the estimate

$$
\sum_{i=0}^{N} \operatorname{ord}_{z=z_{i}} \Theta_{i}(z) \leqslant 8(L+1)(M+1)+2 M N .
$$

Proof. See Brownawell and Masser [1, Theorem 1].

We will also need to bound the modulus of these nonzero values of our auxiliary function. This we achieve through an application of Lemma 2 and the following lemma. 
Lemma 4 (SchWARZ'S LemMA). Let $R>2 r$ be positive numbers and $f$ a nonzero function of one variable which is continuous in the disc $|z| \leqslant R$ and analytic inside. We let $V_{f}(0, r)$ denote the number of zeros (counting multiplicities) of $f$ in the disc $|z|<r$. Then

$$
\log |f|_{r} \leqslant \log |f|_{R}-V_{f}(0, r) \log (R / 2 r),
$$

where $|f|_{r}=\sup _{z=r}|f(z)|$.

Proof. See Waldschmidt [5, Lemma 1.3.1, p. 1.10].

Finally, for any number field $F$ we define the $F$-height of a polynomial $P(X)=$ $\sum_{i=0}^{d} a_{i} X^{i}$ by

$$
h_{F}(P)=\sum_{\nu} N_{\nu} \log \max \left(1,\left|a_{0}\right|_{\nu}, \ldots,\left|a_{d}\right|_{\nu}\right),
$$

where the sum is over all normalized valuations $\nu$ of $F$ over $\mathbf{Q}$, and $N_{\nu}$ denotes the local degree of $\nu$. For a nonzero element $\alpha$ of $F$ we put $h_{F}(\alpha)=\Sigma_{\nu} N_{\nu} \log \max \left(1,|\alpha|_{\nu}\right)$. We then have the fundamental inequality:

Lemma 5. For $\alpha \in F^{*},-h_{F}(\alpha)<\log |\alpha|$.

Proof. By the product formula, $1=\prod_{\nu}|\alpha|_{\nu}^{N_{\nu}}$, we have

$$
0=\sum_{\nu} N_{\nu} \log |\alpha|_{\nu}<\log |\alpha|+\sum_{\nu} N_{\nu} \log \max \left(1,|\alpha|_{\nu}\right)=\log |\alpha|+h_{F}(\alpha) .
$$

Hence $-h_{F}(\alpha)<\log |\alpha|$.

This lemma will be used to produce the desired lower bound for $|P(\wp(\beta u))|$.

In all that follows we take $F=\mathbf{Q}\left(g_{2}, g_{3}, \wp(u), \wp^{\prime}(u)\right)$.

Proof (Theorem A). Let $P(X)$ be an integral polynomial with $d=\operatorname{deg} P$ and $h_{F}=h_{F}(P)$ satisfying $d \geqslant 1, h_{F} \geqslant 0$. Assume for the moment that $P(X)$ is monic and irreducible over $F$. The general case will follow easily from this. Notice that if $P(X)=X$ then it is obvious that there exists a constant $C$ as above satisfying $\log |\wp(\beta u)|>-C$.

Take $C_{1}$ to be a sufficiently large constant and define parameters

$$
\begin{gathered}
D=\left[C_{1}^{9} d t(1+\log t)^{2}\right], \quad K=\left[C_{1}^{13} d t^{2}(1+\log t)^{3}\right], \\
S=\left[C_{1}^{2} d^{1 / 2}(1+\log t)^{1 / 2}\right]
\end{gathered}
$$

where $t=d+h_{F}$.

We will use an auxiliary function of the form

$$
\Phi(z)=\sum_{l=0}^{D} \sum_{m=0}^{D} P_{l m}(\wp(\beta u))(\wp(z))^{l}(\wp(\beta z))^{m}
$$

with the polynomials $P_{l m}$ determined below so that

$$
\Phi^{(k)}(s u)=0 \text { for } 0 \leqslant k \leqslant K, s \in \mathcal{O}^{\prime}(S) .
$$

There are standard polynomials $A_{s}, B_{s}$ such that $\wp(s z)=A_{s}(\wp(z)) / B_{s}(\wp(z))$, and we use these to define "denominators" for our auxiliary function as

$$
\Delta_{s}(z)=\left\{B_{s}(\wp(z)) B_{s}(\wp(\beta z))\right\}^{D} .
$$


We also use the notation $\Theta_{s}(z)=\Delta_{s}(z) \Phi(s z)$. Then if we express each monomial of $\Theta_{s}(z)$ as

$$
\Gamma_{s l m}(z)=\Delta_{s}(z)(\wp(s z))^{l}(\wp(\beta s z))^{m}
$$

we have

$$
\Theta_{s}(z)=\sum_{l=0}^{D} \sum_{m=0}^{D} P_{l m}(\wp(\beta u)) \Gamma_{s l m}(z) .
$$

By the Anderson-Baker-Coates Lemma [5, Lemma 6.2.3] there exist polynomials $G_{s / m}^{k}(X, Y)$ satisfying

$$
\Gamma_{s l m}^{(k)}(u)=G_{s l m}^{k}\left(\wp(\beta u), \wp^{\prime}(\beta u)\right)
$$

In this context $\operatorname{deg}_{\wp_{(\beta u)}} G_{s l m}^{k} \leqslant D S^{2}$ and $\operatorname{deg}_{\wp^{\prime}(\beta u)} G_{s l m}^{k} \leqslant 1$; and the coefficients of $G_{s l m}^{k}$ are polynomial expressions in $\wp(u)$ (of degree $\left.\leqslant D S^{2}\right)$, in $\wp^{\prime}(u)$ (of degree $\leqslant 1$ ), in $\beta$ (of degree $\leqslant K$ ) with coefficients integers in $\mathbf{Q}\left(g_{2}, g_{3}\right)$ with $F$-heights $<C_{3}\left(D S^{2}+K \log K\right)$.

Setting

$$
Q_{s}^{k}(X, Y)=\sum_{l=0}^{D} \sum_{m=0}^{D} P_{l m}(X) G_{s l m}^{k}(X, Y)
$$

for $0 \leqslant k \leqslant K, s \in \mathcal{O}^{\prime}(S)$, we then take $\delta$ to be a denominator for all of $\wp(u), \wp^{\prime}(u)$, and $\beta$ and consider the system of equations

$$
\delta^{D S^{2}+K} Q_{s}^{k}(X, Y)=0 \text { for } 0 \leqslant k \leqslant K \text { and } s \in \theta^{\prime}(S) .
$$

In this situation the coefficients of our unknowns are algebraic integers and we can therefore obtain solutions $P_{l m}(X) \in Z[X]$. Further, since we have $(D+1)^{2}$ unknowns and $(K+1)(S+1)^{2}$ equations, we can solve this system with $P_{l m}(X)$ satisfying $\operatorname{deg} P_{l m}(X) \leqslant D S^{2}$ and $\operatorname{loght}\left(P_{l m}\right) \leqslant C_{5}\left(D S^{2}+K \log K\right)$. Therefore a simple computation yields

$$
\operatorname{deg}_{X} Q_{s}^{k}(X, Y) \leqslant C_{6} D S^{2}, \quad \operatorname{deg}_{Y} Q_{s}^{k}(X, Y) \leqslant 1,
$$

and

$$
h_{F}\left(Q_{s}^{k}(X, Y)\right) \leqslant C_{6}\left(D S^{2}+K \log K\right) .
$$

Notice that, by construction, $Q_{S}^{k}(\wp(u), \wp(\beta u))=\Theta_{s}^{(k)}(u)$; then, since $\Delta_{s}(u) \neq 0$, we deduce that $\Phi^{(k)}(s u)=0\left(0 \leqslant k \leqslant K, s \in \theta^{\prime}(S)\right)$. (It is immediate that $\Delta_{s}(u) \neq 0$ by our assumption that $u$ is a nontorsion point for $\wp$.)

The function $T(z)=(\sigma(z) \sigma(\beta z))^{C_{6} D S^{2}} \Phi(z)$ is entire, therefore Schwarz's Lemma applied to circles of radii $r=C S$ and $R=4 C S$ allows us to majorize $T^{(k)}(s u)$ as $\log \left|T^{(k)}(s u)\right| \leqslant-C_{7} K S^{2}$ for $0 \leqslant k<C^{4} K$ and $s \in \mathcal{O}^{\prime}(S)$. Then, by Lemma 2 we have a lower bound of the form

$$
\log \left|(d / d z)^{k}[\sigma(z) \sigma(\beta z)]^{C_{6} D S^{2}}\right|_{z=u} \geqslant-C_{8} D S^{4}
$$

and, hence, we may conclude that

$$
\log \left|\Phi^{(k)}(s u)\right| \leqslant-C_{9} K S^{2} \quad\left(0 \leqslant k<C^{4} K, s \in \mathcal{O}^{\prime}(S)\right) .
$$


From this we estimate $\left|Q_{s}^{k}\left(\wp(\beta u), \wp^{\prime}(\beta u)\right)\right|$ by observing that

$$
Q_{s}^{k}\left(\wp(\beta u), \wp^{\prime}(\beta u)\right)=(d / d z)^{k}\left(\Delta_{s}(z) \Phi(s z)\right)_{z=u},
$$

and that for $0 \leqslant k<C^{4} K, \log \left|\Delta_{s}^{k}(u)\right| \leqslant C_{10} K \log K$. This, together with the usual estimate for the binomial coefficients, yields

$$
\log \left|Q_{s}^{k}\left(\wp(\beta u), \wp^{\prime}(\beta u)\right)\right| \leqslant-C_{11} K S^{2} \quad \text { for } 0 \leqslant k<C^{4} K \text { and } s \in \mathcal{C}^{\prime}(S) .
$$

If the polynomials $P_{l m}(X)$ have a common factor, $Q(X) \in Z[X]$, then we rewrite our auxiliary function as

$$
\Phi(z)=Q(\wp(\beta u)) \sum_{l, m} \bar{P}_{l m}(\wp(\beta u))(\wp(z))^{l}(\wp(\beta z))^{m},
$$

where the polynomials $\bar{P}_{l m}(X)$ have no common factor.

By Lemma II, p. 135 of Gelfond [2] and the fact that $Q(X) \in Z[X]$ and, therefore $\operatorname{ht}(Q) \geqslant 1$, we have the estimates $\operatorname{deg} \bar{P}_{l m} \leqslant C_{12} D S^{2}$ and $\operatorname{loght} \bar{P}_{l m} \leqslant$ $C_{12}\left(D S^{2}+K \log K\right)$. Since these estimates are essentially the same as for the polynomials $P_{l m}(X)$ we may as well assume that the polynomials $P_{l m}(X)$ have no common factor.

Naturally $Q_{s}^{k}\left(\wp(\beta u), \wp^{\prime}(\beta u)\right)$ is possibly linear in $\wp^{\prime}(\beta u)$. To avoid any complications arising from this fact we eliminate $\wp^{\prime}(\beta u)$ by taking the relative norm of our expression from $F\left(\wp(\beta u), \wp^{\prime}(\beta u)\right)$ to $F(\wp(\beta u))$. These new expressions $\bar{Q}_{s}^{k}(\wp(\beta u))$ satisfy the same estimates with new constants.

Next, suppose that one of the polynomials $\bar{Q}_{s}^{k}(X)$ is prime to $P(X)$; we show below that this must be the case. Forming the resultant of these two polynomials with respect to $X$ and applying Lemma 5, we obtain

$$
\log |P(\wp(\beta u))|>-c d K \log K ;
$$

recalling the definitions of our parameters, we are done.

If, however, none of the polynomials $\bar{Q}_{s}^{k}(X)$ is prime to $P(X)$, then our assumption that $P(X)$ is irreducible implies that $P(X)$ must divide each $\bar{Q}_{s}^{k}$. Then choose $\theta \in C$ such that $P(\theta)=0$, and $\alpha \in C$ such that $\wp(\alpha)=\theta$. Notice that $\bar{Q}_{s}^{k}(\theta)=0$ for all $k$ and $s$ as above.

We now exhibit functions $\Phi_{s}(z)$ which violate the zeros estimate of Lemma 3. Let

$$
\Phi_{s}(z)=\sum_{l=0}^{D} \sum_{m=0}^{D} P_{l m}(\wp(\alpha))(\wp(z))^{l}\left(\wp\left(\beta z+\delta_{s}\right)\right)^{m}
$$

where $\delta_{1}=\alpha-\beta u$ and $\delta_{s}=s \delta_{1}$. Our goal is to show that $\Phi_{s}^{(k)}(s u)=0$ for $0 \leqslant k \leqslant$ $C^{3} K$.

To see this, set

$$
\bar{\Delta}_{s}(z)=\left\{B_{s}(\wp(z)) B_{s}\left(\wp\left(\beta z+\delta_{s}\right)\right)\right\}^{D}
$$

and

$$
\bar{\Gamma}_{s / m}(z)=\bar{\Delta}_{s}(z)(\wp(s z))^{l}\left(\wp\left(s \beta z+\delta_{s}\right)\right)^{m}
$$


Then if $\Theta_{s}^{(k)}(z)=\bar{\Delta}_{s}(z) \Phi_{s}(s z)$, we have the simple relationship:

$$
\Theta_{s}(u)=\sum_{l=0}^{D} \sum_{m=0}^{D} P_{l m}(\wp(\alpha)) \bar{\Gamma}_{s / m}(u) .
$$

From this we next show that $\Theta_{s}^{(k)}(u)=0$ for $0 \leqslant k \leqslant C^{3} K$ and $s \in \Theta^{\prime}(S)$.

As in the construction of our auxiliary function we use the polynomials $G_{s / m}^{k}$ to express the derivatives of $\bar{\Gamma}_{s l m}^{(k)}(z)$, that is, $\bar{\Gamma}_{s / m}^{(k)}(u)=G_{s / m}^{k}\left(\wp(\alpha u), \wp^{\prime}(\alpha u)\right)$. Therefore $\bar{Q}_{s}^{k}(\wp(\alpha))=\Theta_{s}^{(k)}(u)$, which implies that

$$
\Phi_{s}^{(k)}(s u)=0 \text { for } 0 \leqslant k \leqslant C^{3} K \text { and } s \in \mathfrak{C}^{\prime}(S) .
$$

The functions $\Phi_{s}(z)$ are of the type appearing in Lemma 3, therefore (provided that none of them vanishes identically) the total order of zeros at the points $s u$, $s \in \theta^{\prime}(S)$, is bounded by $8(D+1)+\frac{2}{3} D S^{2}$. However, (4) implies that we have at least $\frac{1}{3} C^{3} K S^{2}$ zeros (counting multiplicities) at these points. For our choice of parameters these bounds are contradictory.

Therefore unless some $\Phi_{s}(z)$ vanishes identically, $P(X)$ must be prime to one of the polynomials $\bar{Q}_{s}^{k}(X)$ and our lower bound holds. If one of the functions $\Phi_{s}(z)$ vanishes identically, then we have an algebraic dependence between the functions $\wp(z)$ and $\wp\left(\beta z+\delta_{s}\right)$, and hence between $\wp(z)$ and $\wp(\beta z)$. Therefore there exists a nonzero integer $t$ such that $t \beta \Omega \subseteq \Omega$, and therefore $t \beta \omega_{2}=n \omega_{1}+m \omega_{2}$. This implies that $\beta$ is linear over $K_{\tau}$, contrary to our hypothesis.

We have demonstrated the desired minorization for $|P(\wp(\beta u))|$ when $P(X)$ is irreducible over $F[X]$. For the general case take $P(X) \in Z[X]$ and put $Q(X)=$ $a^{-1} P(X)$ where $a$ is the leading coefficient of $P(X)$. We then factor $Q(X)$ over $F[X]$ as $Q(X)=\prod_{i=0}^{k} P_{i}(X)$ where each polynomial $P_{i}(X)$ is nonconstant, monic and irreducible. If we let $d_{i}=\operatorname{deg} P$ and $t_{i}=\left(d_{i}+h_{F}\left(P_{i}\right)\right)$, then by our first result,

$$
\log \left|P_{i}(\wp(\beta u))\right| \geqslant-C d_{i}^{2} t_{i}^{2}\left(\log t_{i}\right)^{4} \quad \text { for } 1 \leqslant i \leqslant k .
$$

Hence

$$
|\log P(\wp(\beta u))| \geqslant \log |Q(\wp(\beta u))|>-C \sum_{i=0}^{k} d_{i}^{2} t_{i}^{2}\left(\log t_{i}\right)^{4},
$$

and by the usual theory of polynomial heights (e.g. Lang [3, p. 57]),

$$
\sum_{i=0}^{k} d_{i}^{2} t_{i}^{2}\left(\log t_{i}\right)^{4} \leqslant C d^{2} t^{2}(\log t)^{4}
$$

This completes our proof of result $A$.

REMARKS ON THE PROOF OF THEOREM B. Without the hypothesis of complex multiplication we have fewer multiplications with which to extrapolate. In this case, the size of the zeros with which are extrapolate must be enlarged accordingly. This is achieved through the following choice of parameters: $D=\left[C^{13} d^{3} t(1+\log t)^{7}\right]$, $S=\left[C^{3} d(1+\log t)\right], K=\left[C^{22} d^{5} t^{2}(1+\log t)^{13}\right]$. Using these the proof of $\mathrm{B}$ is similar to that of $A$; therefore, we omit the proof of this result. 


\section{REFERENCES}

1. W. D. Brownawell and D. W. Masser, Multiplicity estimates for analytic functions. I, J. Reine Angew. Math. 314 (1980), 200-216.

2. A. O. Gelfond, Transcendental and algebraic numbers, Dover, New York, 1960 (Russian ed., 1951).

3. S. Lang, Introduction to transcendental numbers, Addison-Wesley, Reading, Mass., 1966.

4. D. W. Masser, Elliptic functions and transcendence, Lecture Notes in Math., vol. 437, Springer-Verlag, New York, 1975.

5. M. Waldschmidt, Transcendence methods, Queen's Papers in Pure and Appl. Math., No. 52, Kingston, Ont., 1979.

Department of Mathematics, Pitzer College, Claremont, California 91711 